\title{
Simple and Compact Electrostatic Cs-Corrector using Annular and Circular Electrodes
}

\author{
Tadahiro Kawasaki ${ }^{1,2}$, Takafumi Ishida ${ }^{2}$, Tetsuji Kodama ${ }^{3}$, Masahiro Tomita ${ }^{4}$, Takaomi Matsutani ${ }^{5}$, \\ and Takashi Ikuta ${ }^{6}$, \\ 1. Nanostructures Research Laboratory, Japan Fine Ceramics Center, Nagoya, Japan \\ 2. Institute of Materials and Systems for Sustainability, Nagoya University, Nagoya, Japan \\ 3. Graduate School of Science \& Technology, Meijo University, Nagoya, Japan \\ 4. Vacuum Device Ltd., Mito, Japan \\ 5. Faculty of Science and Engineering, Kinki University, Higashiosaka, Japan \\ 6. Faculty of Engineering, Osaka Electro-Communication University, Neyagawa, Japan
}

It has been well known that the spherical aberration (Cs) has to be compensated to improve the spatial resolution in the electron microscopes. Some types of aberration correction devices have been proposed and developed, and the Cs-correctors consisted of multi-pole lenses have successfully realized sub-angstrom resolution in (S)TEMs [1-3]. However, these correctors require complex control of multiple optical components with high accuracy and stability. They also demand reconfiguration of the microscope columns to insert rather large additional components, resulting in huge cost. In order to solve these problems, one of the coauthor Ikuta had proposed the very simple and compact Cs-corrector with axially-symmetric electrostatic-filed formed between annular and circular electrodes [4], as schematically shown in Fig. 1(a).

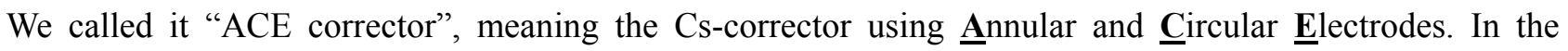
present paper, we report preliminary results of the ACE corrector installed in the STEM apparatus.

Principle of the ACE corrector can be simply explained as follows. Fig. 1(b) shows an example of equipotential lines of the electrostatic field in the cross-section obtained by numerical simulations. In the field around the circular electrode (left-hand side in (b)), the electrons going through the field from left- to right-hand side are a little focused. In contrast, around the annular electrode, the electron trajectories are spread, as shown in Fig. 1(c). They indicate that the electrostatic field formed by the ACE corrector can provide the compound lens effect of the convex and concave lenses arising from the circular and annular electrodes, respectively. Totally, as schematically shown in Fig. 1(d), the $\mathrm{ACE}$ corrector has the negative Cs value, though the effective area is restricted to be in the off-axis by the annular slit.

Fig. 2(a) is a cross-sectional illustration of the electrodes with typical sizes. The circular electrode can be easily obtained by the photolithography as well as the conventional apertures for the electron microscopes. Since the annular electrodes contain complicated structures, we have employed the focused ion beam (FIB) technique for their fabrication. Fig. 2(b) shows a SEM image of the annular slit corresponding to that in Fig. 2(a). This structure was processed at the center of the base tantalum plate having the size of $3 \mathrm{~mm}$ in diameter and $10 \mu \mathrm{m}$ in thickness. Two electrodes were assembled in 
the small device, as shown in Fig. 2(c), by sandwiching the insulator film between them. This device was installed in the STEM (Hitachi HD-2300S; 200kV) by attaching to the tip of the conventional aperture holder instrument, which were connected to the voltage supply. The constant negative voltage was applied to the circular electrode, and the annular electrode was grounded, via two lines attached to the device as in Fig. 2(c).

Figs. 3 show annular dark-field (ADF) images of $\mathrm{CeO}_{2}$ particles taken at different $\mathrm{Cs}$ conditions, i.e. the voltage applied to the ACE corrector varied from $0 \mathrm{~V}$ to $20 \mathrm{~V}$. They indicate that the image obtained at $10 \mathrm{~V}$ show most clear contrast, which is consistent with the appropriate value predicted in advance by the simulation. These results demonstrate that our developed electrostatic device can effectively correct the intrinsic spherical-aberration of the objective lens.

\section{$\underline{\text { References }}$}

[1] H. Rose, Optik 85 (1990) 19-24 [3] O. L. Krivanek, et al., Inst. Phys. Conf., 153 (1997) 35

[2] M. Haider, et al., Optik 99 (1995) 167 [4] T. Kawasaki, et al., Proc. ALC, 27p-P-58, (2015)

(a)

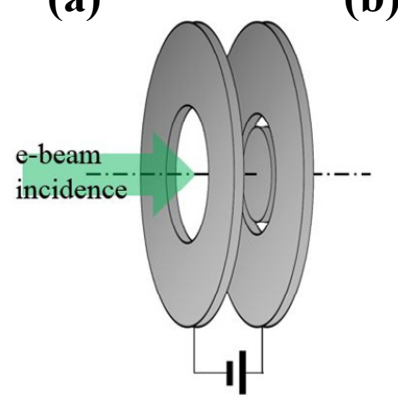

(b)

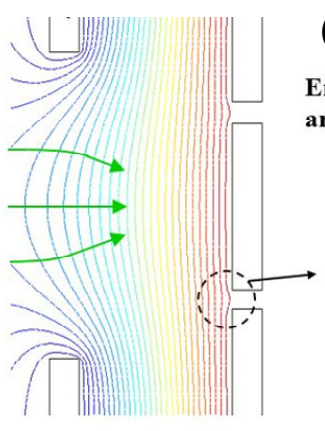

(c)

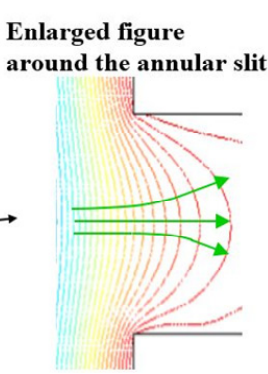

(d)

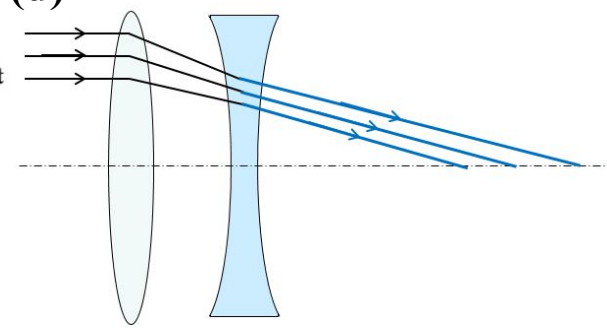

Figure 1. Schematic illustrations of the ACE corrector with equipotential lines inside.

(a)

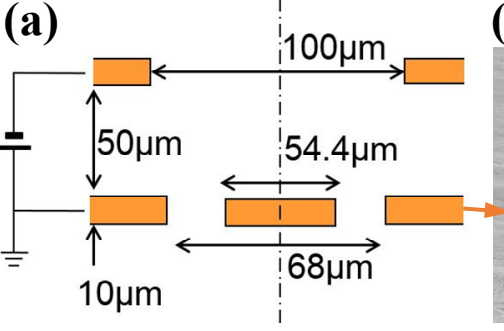

(b)

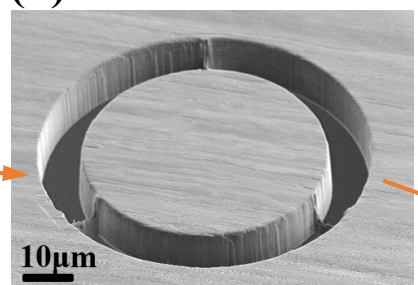

(c)

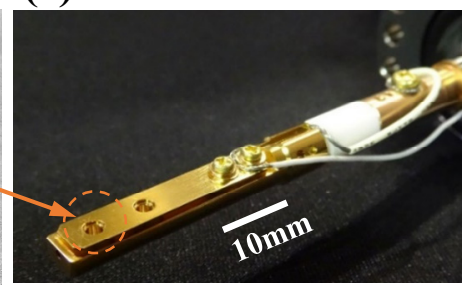

Figure 2. Developed ACE corrector device including the annular electrode.
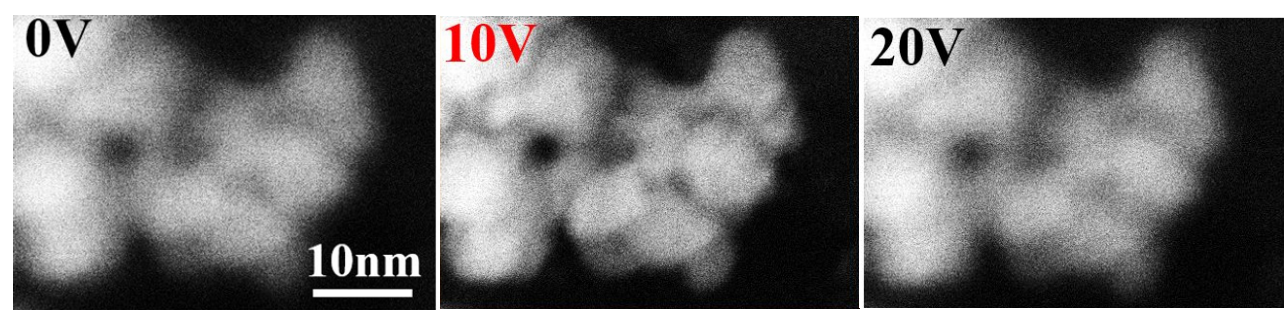

Figure 3. ADF STEM images of $\mathrm{CeO}_{2}$ particles with $\mathrm{ACE}$ corrector. 